\title{
Percepción de una muestra de educandos y docentes sobre la implementación del programa educación para la afectividad y la sexualidad integral
}

\section{Sample of Learners and Teachers Perception on the Implementation of the Affectivity and Integral Sexuality in the Costa Rican Educational Program}

Giselle León León ${ }^{7}$ División de Educología, del Centro de Investigación en Educación

Universidad Nacional

Heredia, Costa Rica leongiselle@hotmail.com

Grettel Bolaños Bolaños ${ }^{2}$ Enseñanza de las Ciencias, Universidad Nacional Heredia, Costa Rica gretel130790@hotmail.com

Johanna Campos Granados 3 Enseñanza de las Ciencias, Universidad Nacional

Heredia, Costa Rica jocagra04@hotmail.com

Fabiola Mejías Rodríguez ${ }^{4}$ Enseñanza de las Ciencias, Universidad Nacional

Heredia, Costa Rica fmr_2289@hotmail.com

Recibido 12 de enero de 2013 • Corregido 08 de marzo de 2013 • Aceptado 13 de marzo de 2013

1 Académica de la División de Educología, Universidad Nacional de Costa Rica. Máster en Administración Educativa, bachiller en la Enseñanza de las Ciencias y licenciada en Ciencias de la Educación con Énfasis en Didáctica de Ciencias. Estudiante del Doctorado en Educación de la Universidad Autónoma de Durango, México.

2 Estudiante de Enseñanza de las Ciencias, Universidad Nacional.

3 Estudiante de Enseñanza de las Ciencias, Universidad Nacional.

4 Estudiante de Enseñanza de las Ciencias, Universidad Nacional. 
URL: http://www.una.ac.cr/educare

CORREO: educare@una.cr

Resumen. La investigación versa sobre la pertinencia de la implementación del programa Educación para la Afectividad y la Sexualidad Integral (EASI) propuesto por el Ministerio de Educación Pública de Costa Rica (MEP), a partir del año 2013. Esta se realizó con el enfoque mixto, en la clasificación de mixto dominante. Para recopilar la información de las categorías del estudio (nivel de conocimiento del tema, modelos de enseñanza y estrategias metodológicas), se aplicó un cuestionario a diecinueve estudiantes (11 de sétimo y 8 de noveno año) y se realizaron entrevistas semiestructuradas a 5 docentes de enseñanza de las ciencias de un colegio diurno del área metropolitana costarricense. Algunos de los aportes fueron resaltados en matrices (tablas) y contrastados mediante la triangulación de informantes y teoría. Dentro de las principales conclusiones se destaca la pertinencia de los programas de educación para la efectividad y sexualidad integral, en opinión de las personas profesionales en educación y de la población estudiantil del estudio. Además, se identificó que el conocimiento de las personas participantes del estudio (estudiantes y docentes) es meramente biológico.

Palabras claves. Educación sexual, programa de estudio, educación secundaria, enseñanza de las ciencias.

Abstract. The case study focuses on the relevance of the Affectivity and Integral Sexuality in the Costa Rican Educational Program (EAIS) implementation. This program was proposed by the Ministry of Public Education (MEP) in 2013. It was implemented using a mixed approach under a dominant mixed classification. To gather data for the different study categories (knowledge of the topic, teaching models, and methodological strategies), a questionnaire was applied to nineteen students and some semi-structured interviews were carried out for five Science teachers from a high school within the Costa Rican Metropolitan Area. Some contributions were emphasized in matrices (charts) and contrasted through of triangulation both of theory and participants. Within the main conclusions stands out the relevance of programs for the effectiveness and integral sexuality supported by professionals in education and the learning population of the study. In addition, it was identified that the participants' 'knowledge of the study (learners and instructors) was merely biological oriented.

Keywords. Sexual education, training program, secondary education, science education.

En las instituciones educativas de Costa Rica se ha evidenciado la necesidad de abordar la temática "salud sexual integral y afectiva" en la formación de adolescentes que cursan el III ciclo de la educación general básica, debido a que tanto en el seno familiar como en el ámbito escolar, existe poca educación al respecto, ya que si bien se aborda, se da prioridad al componente biológico y se deja de lado la integralidad de la temática; no obstante lo indicado por Fallas, Artavia y Gamboa (2012), acerca de que "la sexualidad es una dimensión que se mantiene desde el instante de la concepción hasta la muerte (...)" (p. 54).

Desde esa perspectiva, la educación sexual debe enfocarse como un proceso dinámico, continuo, que conlleve a un desarrollo integral del ser humano, para contribuir con una sana formación e inserción a su entorno. Se concibe como un medio para mejorar las condiciones de vida de los jóvenes y las jóvenes, debido a que puede contribuir a prevenir problemas de salud física, psíquica y madurez emocional, así como a mejorar la convivencia familiar y social. 
En nuestros días, el tema de la educación sexual es cada vez más aceptado en niveles técnicos y pedagógicos, sin embargo, se discute si la manera más adecuada para desarrollar esta temática es mediante una perspectiva meramente biológica y fisiológica, o desde un punto de vista más integral, lo que genera diversos modos de concebir la educación sexual. Por tal motivo, los programas de estudio propuestos por el Ministerio de Educación Pública (MEP) de Costa Rica pretenden promover una visión integral; que permita al estudiante desarrollar, comprender y educarse para la afectividad y sexualidad integral en la vida diaria de la persona adolescente y fomentar, a su vez, la construcción de mejores relaciones entre las personas; todo lo cual repercutirá en su de proceso de madurez.

Lo antes indicado responde a una necesidad tanto nacional como internacional, pues como lo expone la Organización de las Naciones Unidas para la Educación la Ciencia y la Cultura (UNESCO) (2010, p. iii):

En la actualidad, son muy pocas las personas jóvenes que reciben una adecuada preparación para la vida sexual. Este hecho los hace potencialmente vulnerables frente a la coerción, el abuso, la explotación, el embarazo no planificado y las infecciones de transmisión sexual (ITS), incluyendo el VIH.

Ante este panorama, resulta indispensable el desarrollo de estudios que den a conocer la pertinencia de una adecuada formación sexual. Por esto, se han querido compartir los resultados de una investigación realizada en el II ciclo del año 2012, como parte de las actividades del curso Seminario de innovación y producción, previo a la puesta en marcha de los nuevos programas propuestos por el MEP, en una muestra de docentes de secundaria y educandos costarricenses, con el fin de conocer la necesidad de formación en educación sexual de las partes involucradas, a la luz del programa propuesto por el MEP.

\section{Antecedentes}

Se han llevado a cabo varios estudios a nivel nacional acerca de cómo la educación sexual se ha trabajado dentro del marco de la enseñanza. Entre ellos se puede mencionar el trabajo realizado por Cauich (2005), quien señala:

La educación sexual situada dentro del marco de enseñanza de los temas transversales no forma parte de los intereses de la administración y ni de la identidad del profesorado de Educación Secundaria en general; lo que imposibilita su inclusión real y efectiva en el curriculum de la Educación Secundaria y hace que se mantenga al margen de los planes de estudio y seguir en la fila como "asignatura pendiente". (p. 5) 
URL: http://www.una.ac.cr/educare

CORREO: educare@una.cr

Por otro lado, Barros (2001), citado por Zárate (2003), señala que la mayoría de las personas que comienzan las prácticas sexuales precoces son los adolescentes varones, debido a los ideales machistas que tienden a estimular la iniciación sexual temprana y la promiscuidad.

La Universidad Nacional (UNA), a través del Instituto de Estudios Sociales en Población (IDESPO) y el Instituto de Estudios Interdisciplinarios de la Niñez y la Adolescencia (INEINA), llevó a cabo, en el año 2009, en coordinación con el MEP, la propuesta del Plan Nacional de Educación para la Sexualidad, el cual tomó en cuenta la opinión de distintas poblaciones, como niñas y niños, adolescentes, padres y madres de familia, docentes de primaria y secundaria, consultados en una muestra aleatoria a nivel nacional, con el objetivo de conocer la situación de los estudiantes de Costa Rica en el tema de la educación sexual (Arce et al., 2009).

De igual manera se puede indicar el aporte de Fallas (2012) sobre el abordaje de la educación afectiva y sexual en Costa Rica, específicamente en uno de sus ejes conductores: los elementos que deben ser fortalecidos para que el educador o la educadora pueda abordar la educación afectiva y sexual desde el modelo biográfico y profesional. Dentro de las principales conclusiones se destaca que "(...) la acción docente se convierte en un recurso esencial para la respuesta asertiva en la promoción de la salud en general (...)" (p. 614).

\section{Problematización}

¿Cómo analizar la pertinencia de la implementación de los programas de educación para la afectividad y sexualidad integral propuestos por el Ministerio de Educación Pública (MEP), en una sección de sétimo y noveno año en un colegio de la provincia ${ }^{5}$ de San José?

\section{Objetivo general}

Analizar la pertinencia de la implementación de los programas de educación para la efectividad y sexualidad integral, en una sección de sétimo y noveno año en un colegio de la provincia de San José.

\section{Objetivos específicos}

- Identificar el nivel de conocimiento que posee el docente y la docente de secundaria de las secciones 7-A y 9-B en el abordaje de los temas de educación sexual, a la luz de los programas propuestos por el MEP.

5 Corresponde a una organización territorial, para efectos de administración pública. Las cuales son 7: San José, Alajuela, Cartago, Heredia, Guanacaste, Puntarenas y Limón. 
- Reconocer los conocimientos previos sobre educación sexual que tienen los adolescentes de las secciones 7-A y 9-B y la disponibilidad de aumentarlos.

- Identificar los modelos de enseñanza, que prefiere el estudiantado de las secciones 7-A y 9-B del estudio, para desarrollar la temática de educación sexual en su proceso de aprendizaje.

- Describir las estrategias metodológicas que el profesorado utiliza para el abordaje de los temas de educación sexual establecidos para el III Ciclo.

\section{Marco teórico}

\section{Modelos de enseñanza}

Un modelo, según Joyce y Weil (2002), se puede describir como "(...) el conjunto de herramientas que se proporcionan al estudiante para que se enfrente a una tarea de aprendizaje" (p. 36), por lo tanto, los modelos pedagógicos permiten obtener una estrategia donde se pueden validar los procesos de aprendizaje, siempre y cuando estos sean evaluados constantemente, ya que las personas profesionales en educación deben ser conscientes de que cada estudiante presenta un tipo de aprendizaje distinto. Se procura que, mediante estos modelos, la persona estudiante obtenga mayor motivación y asuma un compromiso con la asignatura, y se logre, así, disminuir los niveles de deserción académica y un mejor aprendizaje.

Los modelos de enseñanza se convierten en modelos de aprendizaje porque son una manera de ayudar al estudiantado a obtener información, ideas, habilidades, valores, modos de pensar y medios para expresarse (Joyce y Weil, 2002); lo que les permite aprender con mayor facilidad. Estos modelos deben contar con un fundamento teórico coherente, un respaldo importante tanto desde la investigación académica como de las orientaciones filosóficas, éticas y psicológicas del aprendizaje.

De acuerdo con Pozo (1997), se puede hacer una clasificación de 5 modelos para enseñar ciencias, entre ellos están:

Modelo tradicional: Considerado el más predominante en todos los centros de educación, debido a que la formación estudiantil se fundamenta en el aprendizaje exacto de la disciplina y no en la práctica didáctica, lo que implica una transmisión verbal de los saberes como si fuesen acabados, objetivos, absolutos y verdaderos (Kaufman, citado por Ruiz, 2008). Asimismo, la persona docente es considerada como una distribuidora de conocimientos y contenidos ya elaborados, donde no toma en cuenta el contexto y los factores de aprendizaje de los educandos, de igual forma la persona estudiante es visualizada como un ser pasivo, ignorante, depositario y consumidor de conocimientos (Pozo, 1997).

Modelo por descubrimiento: Según Sarramona (2008), citando a Brurner (1978), el aprendizaje por descubrimiento es un proceso de aprendizaje inductivo, mediante el cual se 
URL: http://www.una.ac.cr/educare

CORREO: educare@una.cr

pretende elaborar conceptos a partir de los que se puede desarrollar, de forma más profunda, la información obtenida durante dicho proceso. Por su parte, Coon (2005) afirma que el aprendizaje por descubrimiento es un proceso que permite que se adquieran destrezas más por discriminación y comprensión, que por una simple mecanización o práctica rutinaria.

Modelo expositivo: Se enfoca principalmente en el discente y en los conocimientos previos que este presenta y, a partir de ellos, se construyen continuamente los saberes propios de la disciplina, es decir, es necesario conocer lo que el educando sabe sobre la materia para luego enseñar a partir de esas ideas (Ruiz, 2008), a pesar de ello sigue siendo un modelo basado en la transmisión, y deja de lado el aprendizaje procedimental y actitudinal (Pozo, 1997).

Modelo de conflicto cognitivo: Surge a partir del modelo expositivo; en este modelo el estudiantado debe estar consciente de sus pre-saberes y limitaciones, también debe comparar la información aprendida con la enseñada en dicho momento, para luego consolidar los temas desconocidos y poder aplicarlos en distintas contextos (Ruiz, 2008). El fin de dicho modelo es que el estudiante y la estudiante construyan su propio conocimiento, logren confrontar y sustituir los saberes previos por otros conceptos o teorías más fuertes (Pozo, 1997).

Modelo por investigación: Comprende la construcción del conocimiento mediante la resolución de problemas aplicados en el entorno de vida. De acuerdo con este modelo, el estudiante y la estudiante son seres dinámicos que conciben no solo conceptos, sino también destrezas, habilidades y actitudes positivas hacia la ciencia; y la persona profesional en educación es una guía y dirigente de la investigación, que plantea a los educandos situaciones representativas y significativas para promover el desarrollo del pensamiento y la búsqueda de soluciones a los acontecimientos nacionales (Ruiz, 2008). No obstante, este modelo conlleva una problemática para el educador y la educadora, pues requiere, constantemente, de cambios en los métodos de enseñanza utilizados (Pozo, 1997).

\section{Estrategias metodológicas}

Todo modelo de enseñanza se encuentra inmerso en la utilización de estrategias de aprendizaje, que a su vez comprenden el uso de recursos, métodos y técnicas didácticas.

Las estrategias de aprendizaje se consideran una guía de las acciones que hay que seguir en los procesos de educación; además, son siempre consecuentes, reflexivas, premeditadas y están dirigidas a un conocimiento, habilidad o actitud que se ha de aprender en un determinado contexto (Monereo, citado en Herrera, 2009). Como recién se mencionó, las estrategias involucran los métodos y técnicas. Los primeros se definen como "(...) el conjunto de momentos y técnicas lógicamente coordinados para dirigir el aprendizaje del alumno hacia determinados objetivos" (Vargas, 1997, p. 140), mientras que las técnicas constituyen "(...) el recurso didáctico al cual se acude para concretar un momento de la lección (...)" (Vargas, 1997, p. 145), por ende, son también formas de orientación del aprendizaje. 


\section{Métodos y técnicas didácticas}

Existen muchas formas de clasificar los métodos y técnicas de aprendizaje, como la propuesta por Jabif (2007), que se describe a continuación:

\section{Métodos de enseñanza:}

1. Proyecto: Es un método de enseñanza integrado que conlleva dejar de lado la enseñanza tradicional para enfocarse en un trabajo más desafiante y complejo, en cuanto requiere conocimiento interdisciplinario; además, se encuentra centrado en la persona estudiante y en las acciones de esta, por lo que las tareas y problemas planteados deben tener cierta afinidad con situaciones reales; comprende las siguientes fases: informar, planificar, decidir, realizar controlar, valorar y reflexionar (Tippelt y Lindemann, 2001).

2. Aprendizaje basado en problemas: El estudiantado resuelve problemas mediante el método científico, con lo que consigue una adquisición de destrezas que generan un pensamiento crítico, productivo, organizado y sistemático, que le permiten interpretar problemas, formular hipótesis y elaborar conclusiones (Vargas, 1997).

3. Trabajos en grupo: Favorecen una mayor interacción entre docente-estudiante y estudiante-estudiante; de igual forma el aprendizaje es activo, constructivo e interpersonal; además, permite el desarrollo de capacidades intelectuales, creativas, comunicativas y el autoconocimiento (Fernández, 2005).

4. Prácticas de laboratorio: Exponen al educando ante una situación práctica, donde tiene contacto con objetos, fenómenos reales o simulados; en el proceso el estudiante y la estudiante elaboran y comprueban hipótesis e interpretan para lograr la resolución de problemas (Vargas, 1997).

5. Aprendizaje autónomo: El educando es un ser activo que toma la iniciativa y experimenta de forma independiente, proponiéndose sus propias metas de aprendizaje, por lo que durante el proceso es más responsable, y desarrolla habilidades y actitudes que le permiten seguir adquiriendo saberes de forma factible y autónoma (Fernández, 2005).

6. Método expositivo: Exposición oral por parte de la persona profesional en educación sobre un tema estructurado, debe utilizarse cuando inicia un nuevo tema y requiere de explicaciones conceptuales complejas (Jabif, 2007). 
URL: http://www.una.ac.cr/educare

CORREO: educare@una.cr

\section{Educación para la afectividad y sexualidad integral en el marco de la formación ética, estética y ciudadana}

La educación para la afectividad y sexualidad integral que se promueve con la implementación del programa en su primera fase, en el tercer ciclo de la educación general básica:

(...) está dirigida hacia la construcción del vínculo humano desde las dimensiones afectiva, corporal y espiritual, y en marco de la promoción de la madurez emocional. En ese sentido, es una visión integral según la cual comprender, educar, conducir adecuadamente este vínculo y desarrollar, al mismo tiempo, la madurez emocional, será de gran impacto positivo en la convivencia humana y con ello sobre la calidad de vida de [sic] los/as estudiantes, las familias y comunidades de nuestro país.

Educar para la afectividad y la sexualidad integral implica formar para la construcción de mejores relaciones entre las personas y esto conlleva principios filosóficos básicos. En primer lugar, el principio de educar por medio del conocimiento crítico de la realidad. Se trata de un proceso educativo que no es una operación meramente intelectual sino también un proceso articulado a la práctica. Implica conocer el mundo desde y en la práctica transformadora, en la cual intervienen deseos, valores, voluntades, emociones, capacidad de disfrute, imaginación, intenciones y responsabilidad.

En segundo lugar, dicha formación se basa en el principio de educar como práctica política. Se parte de que las prácticas siempre son políticas porque involucran valores y proyectos que reproducen, legitiman, cuestionan o transforman las relaciones de poder prevalecientes en la sociedad. En la educación para la afectividad es de particular importancia la generación de espacios que permitan reducir y evitar la violencia que ha existido dentro de los vínculos humanos, especialmente a nivel de las familias, comunidades y los centros educativos. (República de Costa Rica, MEP, 2012, pp. 2-3)

Como todo proceso de aprendizaje, el planteamiento que realiza el MEP parte de la Política educativa siglo XXI y se fundamenta en tres principios epistemológicos:

(...) la realidad cercana, considerar la educación como parte del proceso de la constitución de las personas y considerar la educación como un diálogo. El primero se refiere a la necesidad de partir del respeto a los valores, principios éticos y las creencias de las personas y las comunidades, con el fin de que la educación sea un proceso de vida en todo momento (...). 
En cuanto a la construcción de la persona como principio orientador (...) implica ofrecer espacios para tomar conciencia, es decir, ordenar el contenido de los sentidos desde los primeros años del desarrollo de las personas, para establecer procesos de reflexión y no solo de información (...).

Finalmente, se considera que educar es diálogo, elemento esencial para poder promover la madurez y plenitud de la sexualidad. La evolución que lleve a la plenitud se dará si se logra establecer una maduración efectiva, una autodeterminación responsable y la claridad para poder dejar actitudes de la cultura del "tener" y poder desarrollar más actitudes de la cultura del "ser", de manera que se convierta en un hilo conductor de lo que es la persona y de su evolución. (República de Costa Rica, MEP, 2012, p. 3)

\section{Ejes temáticos del programa de estudio}

Los ejes temáticos que guían el proceso de mediación pedagógica en el programa educación para la afectividad y sexualidad integral son: "Relaciones interpersonales, cultura, poder y responsabilidad, placer como fuente de bienestar, género, identidad psicosexual, salud reproductiva y derechos humanos" (República de Costa Rica, MEP, 2012, p. 4).

Los ejes temáticos antes mencionados, se proponen por nivel, de manera que en sétimo se aborda el tema conceptos básicos de sexualidad; en octavo, condiciones físicas, emocionales, éticas y culturales en la vivencia de la sexualidad y, en noveno, estrategias para una vivencia sexual saludable, responsable y placentera. En cada caso los temas son tratados a lo largo de los trimestres, acorde al nivel cognitivo y madurez emocional (República de Costa Rica, MEP, 2012).

\section{Importancia de la educación sexual en los adolescentes}

El termino sexualidad en la persona adolescente se convierte en un punto de suma importancia en la enseñanza. A partir del siglo XVIII se agudiza la tarea del educador, donde se desarrolla la tendencia de fomentar lo que se desea de las personas adolescentes y se reprimen las conductas amenazantes para los fines del control social. La sociedad comienza a crear mecanismos de control claramente definidos, como infundir temor y culpa en lo referente a la sexualidad.

La adolescencia es, generalmente, una época de temores, ansiedades y emociones, donde se presentan muchas preocupaciones y angustias que giran alrededor de cuestiones sexuales y físicas, tales como su aspecto personal, desarrollo emocional y corporal. En este sentido, el inicio de la adolescencia encierra muchos secretos para el joven y la joven, debido a que el cuerpo experimenta una diversidad de cambios, percibidos muchas veces por las personas adolescentes como alarmantes. 
URL: http://www.una.ac.cr/educare

CORREO: educare@una.cr

Según Papalia, Wendkos y Duskin (2010), la adolescencia es un período de la vida en el que se produce una serie de cambios físicos, de madurez reproductiva; se da la búsqueda de identidad (incluyendo la sexual), que sitúan al individuo ante una nueva forma de vivenciarse a sí mismo y a todo aquello que le rodea. Esos nuevos cambios hacen una readaptación en todos los sentidos y, por esto, a las personas adolescentes les resulta difícil asimilar las dificultades para encontrarse a sí mismas.

Tomando en cuenta que es una etapa de cambios, y en el marco de una sociedad globalizada donde cada día aumenta la necesidad de información, se hace necesaria una adecuada educación sexual que permita a los adolecentes y a las adolescentes comprender el inmenso valor que tiene el hecho de que cada ser humano pueda darse enteramente a los demás, respetándose a sí mismo y a sus semejantes. Esta educación les ayudará a proyectarse en la sociedad con conocimiento de sus limitaciones y habilidades, y con un sólido y fuerte concepto de sí mismos y una conciencia recta y clara (Ramo, 2008).

\section{Metodología}

\section{Enfoque investigativo}

La presente investigación se ubica dentro del enfoque mixto, a su vez responde a la clasificación de enfoque mixto dominante, sustentado por Hernández, Fernández y Baptista (2006): “(...) el estudio se desarrolla bajo la perspectiva de alguno de los dos enfoques, la cual prevalece, y la investigación mantiene un componente del otro enfoque" (p. 773). En la presente investigación dominó el enfoque cualitativo, con algunos elementos de corte cuantitativo, específicamente en el diseño de instrumentos, como el cuestionario.

El enfoque cualitativo "(...) evalúa el desarrollo natural de los sucesos, es decir, no hay manipulación ni estimulación con respecto a la realidad" (Corbetta, 2003, citado en Hernández, Fernández y Baptista, 2006. p. 9), de igual manera es interpretativo, pues intenta buscar sentido a las situaciones o fenómenos en términos de los significados que las personas le otorguen, sin generalizar puesto que los hallazgos son particulares (Hernández et al., 2006).

La presente investigación responde a ese tipo de enfoque, ya que buscó interpretar el proceso de aprendizaje de la educación sexual, específicamente en la enseñanza de las ciencias. Es decir, se interpretó la opinión de docentes y estudiantes sobre los pros y contras de la propuesta dada por el MEP, en cuanto a la educación para la efectividad y sexualidad integral, desde lo que el estudiantado participante dice requerir y en relación con las opiniones del grupo de docentes en cuanto al conocimiento del tema ${ }^{6}$. Finalmente, se debe agregar que el estudio no pretende generalizar los resultados, sino que estos son propios de esta investigación.

6 El estudio se realizó durante Il ciclo del año 2012, por cuanto los docentes no habían sido capacitados. 


\section{Categorías de análisis}

Al recopilar la información y reflexionar sobre los datos obtenidos, se distinguieron una serie de categorías para el análisis de la información, las cuales se detallan:

1. Nivel de conocimiento: "Es una construcción producto de la actividad del sujeto en su interacción con el medio ambiente físico y social, por lo que convergen en adoptar premisas constructivistas e interaccionistas" (Rodríguez, 1999, p. 481).

2. Modelos de enseñanza: Es un plan estructurado que guía la labor del docente a través de la implementación de diferentes estrategias metodológicas, que comprenden el uso de métodos, técnicas y recursos didácticos (Morán, 2008).

3. Las estrategias metodológicas: Se refieren a los modos ordenados o maneras sincronizadas en que los facilitadores llevarán a la práctica su labor de enseñanza y acompañarán al participante facilitando sus procesos de aprendizaje (Díaz y Hernández, 2002).

\section{Participantes}

La muestra con la que se trabajó en esta investigación estuvo constituida por 11 estudiantes de sétimo y 8 de noveno año, con edades entre 12 y 18 años, de un colegio de la Dirección Regional Educativa de San José. De igual manera, se contó con la colaboración de 5 docentes del área de ciencias pertenecientes a la misma institución.

\section{Instrumentos y técnicas empleados}

Uno de los instrumentos empleados para realizar la investigación fue la entrevista semi-estructurada, aplicada a los cinco docentes participantes de ciencias. La entrevista estaba constituida por 10 preguntas y tenía como propósito fundamental indagar sobre las estrategias metodológicas a las cuales recurrirían para impartir las clases de sexualidad y sobre el manejo del tema.

Por otra parte, se aplicó un cuestionario constituido por 14 preguntas (3 cerradas y 11 abiertas) y destinado al estudiantado de las secciones 7-A y 9-B. Este instrumento se aplicó con el objetivo de indagar sobre los conocimientos previos sobre educación sexual y los modelos de enseñanza que más les gustaría que fueran implementados por los docentes y las docentes en las clases de sexualidad; además, para conocer la opinión sobre los beneficios de una adecuada formación en la temática. Tal como se indicó antes, en el enfoque mixto dominante se puede hacer uso de instrumentos de corte cuantitativo, tal como el cuestionario, si la estructura de la pregunta permite profundizar en el estudio. 
URL: http://www.una.ac.cr/educare

CORREO: educare@una.cr

\section{Consideraciones éticas}

La presente investigación, se realizó con la autorización respectiva del director de la institución, además se contó con el aporte consentido de los docentes y las docentes del Departamento de Ciencias y con la aceptación voluntaria del estudiantado de las secciones 7-A y 9-B para colaborar en el estudio.

\section{Interpretación de resultados}

En este apartado se realiza una descripción detallada de las principales respuestas que se obtuvieron a partir de los instrumentos aplicados.

En primera instancia se presentan los resultados obtenidos del cuestionario aplicado a estudiantes de sétimo, seguido por los de noveno año. Por último, se muestran los resultados del cuestionario aplicado al grupo de docentes de ciencias. Algunos de los aportes fueron resaltados en matrices (tablas) y contrastados mediante la triangulación de informantes y teoría.

\section{Análisis de los resultados del instrumento aplicado a los estudiantes de sétimo año}

Las primeras 5 preguntas permitieron hacer un sondeo sobre elementos como conocer a quién acuden los adolescentes y las adolescentes usualmente, si tienen un problema o duda sobre temas de sexualidad. Tomando como referencia las respuestas, se pudo detectar que la mayoría del estudiantado acude principalmente a la madre de familia, aunque algunos mencionan a sus padres.

En cuanto a los conocimientos sobre sexualidad, refieren tener nociones generales sobre las partes de los órganos reproductores, infecciones de transmisión sexual y métodos de planificación. Estos conocimientos los han adquirido principalmente en la escuela y el colegio, pero consideran que no se han abordado lo suficiente, ya que indican que la metodología utilizada por sus docentes no es la ideal; además de no tener la confianza para abordar dudas específicas del tema. Asimismo, apuntan que algunas personas docentes no le dan la importancia necesaria a esta temática y la abordan de una forma muy superficial, dejando de lado dudas.

En cuanto a los modelos de enseñanza, las personas encuestadas indicaron preferir clases expositivas y centradas en el personal docente; a pesar de que, como lo indican Joyce y Weil (2002), la implementación de estos modelos puede afectar la comprensión del discente, situación que es expuesta por la muestra de educandos de sétimo al aducir tener dudas en el tema, a pesar de su disposición para aprender.

Para seguir ahondando al respecto, se exponen, en la tabla 1, algunas de las respuestas aportadas por el grupo de estudiantes de sétimo, en cuanto a la pertinencia de la implementación de los programas de educación para la afectividad y la sexualidad integral. 
URL: http://www.una.ac.cr/educare CORREO: educare@una.cr

Tabla 1

Relevancia de la implementación de los programas de educación para la afectividad y sexualidad integral, opinión de estudiantes de sétimo nivel

\begin{tabular}{|c|c|c|c|}
\hline Cuestionante & $\begin{array}{l}\text { Modificaciones del } \\
\text { estudiante para mejorar las } \\
\text { clases de educación sexual }\end{array}$ & $\begin{array}{l}\text { Preocupaciones más importantes en } \\
\text { temas de sexualidad }\end{array}$ & $\begin{array}{l}\text { Importancia de la } \\
\text { implementación del programa de } \\
\text { EASI, en los salones de clases. }\end{array}$ \\
\hline $\begin{array}{l}\text { Opinión del } \\
\text { estudiante } 1\end{array}$ & Hablar educadamente & Infecciones de transmisión sexual & Sí, porque aprendemos más \\
\hline $\begin{array}{l}\text { Opinión del } \\
\text { estudiante } 2\end{array}$ & $\begin{array}{l}\text { Tener más información, } \\
\text { para iniciar relaciones } \\
\text { sexuales a edad madura }\end{array}$ & Infecciones de transmisión sexual & Sí \\
\hline $\begin{array}{l}\text { Opinión del } \\
\text { estudiante } 3\end{array}$ & $\begin{array}{l}\text { Que hablen más sobre el } \\
\text { tema }\end{array}$ & $\begin{array}{l}\text { Infecciones de transmisión sexual, } \\
\text { formas de dar placer y formas de } \\
\text { expresar afecto y conciliar diferencias }\end{array}$ & Sí, porque es importante \\
\hline $\begin{array}{l}\text { Opinión del } \\
\text { estudiante } 4\end{array}$ & $\begin{array}{l}\text { Que nos hablen más para } \\
\text { no hacer cosas malas }\end{array}$ & $\begin{array}{l}\text { Infecciones de transmisión sexual, } \\
\text { sexualidad responsable y prevención } \\
\text { de riesgos }\end{array}$ & Sí \\
\hline $\begin{array}{l}\text { Opinión del } \\
\text { estudiante } 5\end{array}$ & $\begin{array}{l}\text { Que nos hablen más del } \\
\text { tema }\end{array}$ & $\begin{array}{l}\text { Infecciones de transmisión sexual, } \\
\text { formas de dar placer y formas de } \\
\text { expresar afecto y conciliar diferencias, } \\
\text { sexualidad responsable, prevención de } \\
\text { riesgos, embarazos }\end{array}$ & No \\
\hline $\begin{array}{l}\text { Opinión del } \\
\text { estudiante } 6\end{array}$ & Todo & Infecciones de transmisión sexual & No, porque no es necesario \\
\hline $\begin{array}{l}\text { Opinión del } \\
\text { estudiante } 7\end{array}$ & $\begin{array}{l}\text { Que nos hablen más del } \\
\text { tema }\end{array}$ & Infecciones de transmisión sexual & Sí \\
\hline $\begin{array}{l}\text { Opinión del } \\
\text { estudiante } 8\end{array}$ & $\begin{array}{l}\text { Que nos hablen más del } \\
\text { tema }\end{array}$ & Infecciones de transmisión sexual & Sí \\
\hline
\end{tabular}

Nota: Resultados obtenidos de los cuestionarios aplicados a 8 estudiantes de secundaria de la sección 7-A.

Como se puede observar en la tabla 1, entre las modificaciones que el grupo de estudiantes propone para mejorar las lecciones de educación sexual están las siguientes: que se les hable más del tema utilizando un lenguaje adecuado, para tener una visión más clara de la temática, lo cual resalta el modelo tradicional centrado en el docente a partir de conocimientos elaborados y deja de lado los conocimientos previos del educando (Pozo, 1997). De igual manera, 100\% dice requerir información adecuada sobre infecciones de transmisión sexual. Dicho esto, se evidencia que la formación actual no es suficiente, contrastando la opinión de los estudiantes y las estudiantes se constata la relevancia de la puesta en marcha de una adecuada formación para la afectividad y sexualidad integral, ya que como se indica a nivel teórico se pretende formar: 
URL: http://www.una.ac.cr/educare

CORREO: educare@una.cr

(...) hacia la construcción del vínculo humano desde las dimensiones afectiva, corporal y espiritual, y en marco de la promoción de la madurez emocional. En ese sentido, es una visión integral según la cual comprender, educar, conducir adecuadamente este vínculo y desarrollar, al mismo tiempo, la madurez emocional, será de gran impacto positivo en la convivencia humana y con ello sobre la calidad de vida de los/as estudiantes, las familias y comunidades de nuestro país. (República de Costa Rica, MEP, 2012, p. 2.)

En opinión de los sujetos encuestados, la sexualidad se resume en sexualidad responsable, infecciones de transmisión sexual y dejan de lado elementos muy importantes como madurez emocional. Además, no visualizan la educación sexual de manera integral, como un componente del ser humano "(...) deseos, valores, voluntades, emociones, capacidad de disfrute, imaginación, intenciones y responsabilidad" (República de Costa Rica, MEP, 2012, p. 2).

\section{Análisis de los resultados del instrumento aplicado a los estudiantes de noveno año}

En esta sección se muestran los resultados obtenidos del cuestionario aplicado a estudiantes de la sección 9-B.

En cuanto a conocer a quién acuden para abordar temas de sexualidad, se pudo determinar que 8 de 11 (73\%) estudiantes acuden a algún familiar cercano como madre o hermanos(as), los 3 restantes señalaron acudir a sus mejores amigos o amigas.

Por otra parte, varias personas de este grupo consideran que las lecciones deben ser más informativas, en donde se les diga de forma directa las consecuencias que trae consigo una vida sexual no responsable. De igual manera afirman que no han desarrollado la confianza suficiente con las personas profesionales de la educación para plantearles las preguntas necesarias. En suma, el grupo de dicentes de noveno, a diferencia de los de sétimo nivel, prefieren modelos más vivenciales, tales como el modelo de conflicto cognitivo; de manera que se logre partir de la realidad inmediata que viven y, en ese caso, poder aplicar los conocimientos en distintos contextos. Asimismo, hacen alusión al modelo por investigación, al proponer charlas con expertos o vivencias de personas (Ruiz, 2008).

En relación con lo expresado por el estudiantado de esta sección, la UNESCO (2010) indica que:

Debemos decidir: o dejamos a los niños y niñas encontrar su propio camino en la nebulosa de información parcial, la desinformación y la explotación que podrían encontrar en los medios de comunicación, Internet, grupos de pares o personas inescrupulosas; o aceptamos la tarea de proporcionar una educación en sexualidad clara, informada, sustentada en hechos (...). (p. iii)

Con respecto al conocimiento sobre sexualidad, este grupo de estudiantes indicó los mismos temas que los de sétimo, asimismo expusieron haberlos abordado en materias como 


\section{ciencias; sin embargo, al igual que los de sétimo nivel, dicen que el abordaje ha sido insuficiente, puesto que expresan que sus dudas no han sido evacuadas.}

Tabla 2

Pertinencia de la implementación de los programas de educación para la afectividad y sexualidad integral, opinión de estudiantes de noveno año

\begin{tabular}{|c|c|c|c|}
\hline Cuestionante & $\begin{array}{l}\text { Modificaciones del estudiante } \\
\text { para mejorar las clases de } \\
\text { educación sexual }\end{array}$ & $\begin{array}{l}\text { Preocupaciones más importantes en temas de } \\
\text { sexualidad }\end{array}$ & $\begin{array}{l}\text { Importancia de la implementación } \\
\text { de las guías de educación para la } \\
\text { afectividad y la sexualidad integral, } \\
\text { en los salones de clases }\end{array}$ \\
\hline $\begin{array}{l}\text { Opinión del } \\
\text { estudiante } 1\end{array}$ & $\begin{array}{l}\text { Que sea una clase en específico, } \\
\text { para la sexualidad solamente. }\end{array}$ & $\begin{array}{l}\text { Infecciones de transmisión sexual y } \\
\text { prevención de riesgos. }\end{array}$ & $\begin{array}{l}\text { Sí, porque así estaremos más } \\
\text { preparados y sabremos lo que } \\
\text { causa bueno y malo de las } \\
\text { relaciones. }\end{array}$ \\
\hline $\begin{array}{l}\text { Opinión del } \\
\text { estudiante } 2\end{array}$ & $\begin{array}{l}\text { Me gustaría que hicieran charla } \\
\text { por nivel para aplicar y dejar } \\
\text { más claro este tema. }\end{array}$ & $\begin{array}{l}\text { Infecciones de transmisión sexual, formas de } \\
\text { expresar afecto y de conciliar diferencias. }\end{array}$ & $\begin{array}{l}\text { Sí, para prevenir embarazos y } \\
\text { enfermedades. }\end{array}$ \\
\hline $\begin{array}{l}\text { Opinión del } \\
\text { estudiante } 3\end{array}$ & $\begin{array}{l}\text { Ver testimonios de personas } \\
\text { con enfermedades y tomar } \\
\text { el ejemplo de ellos para no } \\
\text { cometer el mismo error. }\end{array}$ & $\begin{array}{l}\text { Infecciones de transmisión sexual, sexualidad } \\
\text { responsable y prevención de riesgos. }\end{array}$ & $\begin{array}{l}\text { Sí, ya que se trataría de prevenir } \\
\text { más embarazos a temprana edad. }\end{array}$ \\
\hline $\begin{array}{l}\text { Opinión del } \\
\text { estudiante } 4\end{array}$ & $\begin{array}{l}\text { Que sea una clase especifica y } \\
\text { con responsabilidad. }\end{array}$ & $\begin{array}{l}\text { Infecciones de transmisión sexual, sexualidad } \\
\text { responsable. }\end{array}$ & Sí. \\
\hline $\begin{array}{l}\text { Opinión del } \\
\text { estudiante } 5\end{array}$ & $\begin{array}{l}\text { Qué los profesores hablen más } \\
\text { sobre sexo y como protegernos } \\
\text { de las enfermedades. }\end{array}$ & $\begin{array}{l}\text { Infecciones de transmisión sexual y formas de } \\
\text { dar placer. }\end{array}$ & Sí. \\
\hline $\begin{array}{l}\text { Opinión del } \\
\text { estudiante } 6\end{array}$ & $\begin{array}{l}\text { No sé muchas cosas, para } \\
\text { que no haya embarazos tan } \\
\text { tempranos. }\end{array}$ & $\begin{array}{l}\text { Infecciones de transmisión sexual y quedar } \\
\text { embarazada. }\end{array}$ & Sí, para prevenir muchas cosas. \\
\hline $\begin{array}{l}\text { Opinión del } \\
\text { estudiante } 7\end{array}$ & Deberían dar más charlas. & $\begin{array}{l}\text { Infecciones de transmisión sexual, sexualidad } \\
\text { responsable y prevención de riesgos. }\end{array}$ & $\begin{array}{l}\text { Sí, porque hay muchos } \\
\text { embarazos. }\end{array}$ \\
\hline $\begin{array}{l}\text { Opinión del } \\
\text { estudiante } 8\end{array}$ & $\begin{array}{l}\text { Darles una charla sobre cómo es } \\
\text { la sexualidad. }\end{array}$ & $\begin{array}{l}\text { Infecciones de transmisión sexual, sexualidad } \\
\text { responsable y prevención de riesgos. }\end{array}$ & Sí. \\
\hline $\begin{array}{l}\text { Opinión del } \\
\text { estudiante } 9\end{array}$ & $\begin{array}{l}\text { Un horario donde existan } \\
\text { lecciones especificas para estas } \\
\text { clases. }\end{array}$ & $\begin{array}{l}\text { Formas de expresar afecto y de conciliar } \\
\text { las diferencias, sexualidad responsable y } \\
\text { prevención de riesgos. }\end{array}$ & $\begin{array}{l}\text { Sí, para la prevención y } \\
\text { responsabilidad. }\end{array}$ \\
\hline $\begin{array}{l}\text { Opinión del } \\
\text { estudiante } 10\end{array}$ & $\begin{array}{l}\text { Que las personas que la den } \\
\text { traten de evacuar todas las } \\
\text { dudas posibles y que nos } \\
\text { enseñen bien, de manera activa. }\end{array}$ & $\begin{array}{l}\text { Infecciones de transmisión sexual, formas de } \\
\text { expresar afecto y de conciliar las diferencias, } \\
\text { sexualidad responsable y prevención de } \\
\text { riesgos. }\end{array}$ & Sí, para saber más. \\
\hline $\begin{array}{l}\text { Opinión del } \\
\text { estudiante } 11\end{array}$ & $\begin{array}{l}\text { Ocupar dos lecciones de ciencias } \\
\text { para las clases sexuales. }\end{array}$ & $\begin{array}{l}\text { Infecciones de transmisión sexual, formas de } \\
\text { dar placer y sexualidad responsable. }\end{array}$ & $\begin{array}{l}\text { Sí, para que no se arruinen la vida } \\
\text { con embarazos no deseados. }\end{array}$ \\
\hline
\end{tabular}

Nota: Resultados obtenidos de los cuestionarios aplicados a once estudiantes de secundaria de la sección 9-B. 
URL: http://www.una.ac.cr/educare

CORREO: educare@una.cr

Como se puede observar en la tabla 2, entre las modificaciones propuestas por el estudiantado encuestado para mejorar las lecciones de ciencias, específicamente en el tema de sexualidad, están: la realización de charlas con especialistas que les evacuen las dudas más comunes en esa edad, así como la implementación de una clase exclusiva para hablar de una forma más clara sobre los pros y los contras de una vida sexual temprana, lo cual realza la opinión de modelos pedagógicos más activos, como el conflicto cognitivo, trabajos de campos, entre otros.

En cuanto a las temáticas que se deben abordar en las lecciones de sexualidad, mencionan, al igual que el otro grupo, la sexualidad responsable. Al respecto, expresan que pueden influir las presiones de sus amigos o amigas que ya han tenido este tipo de experiencias y la curiosidad por querer experimentar sensaciones diferentes en su cuerpo, además de la falta de comunicación y atención por parte de algunos padres y madres de familia hacia esta temática, ya que consideran que es un contenido difícil de hablar de una forma abierta con ellos.

Cabe añadir que el 100\% mencionó que es de gran importancia la implementación del programa de educación para la afectividad y la sexualidad integral, ya que es una forma de evitar los embarazos no deseados y prevenir infecciones de transmisión sexual; además porque les permite adoptar una mentalidad más responsable con miras a tener una vida sexual sana y, sobre todo, estar más informados y evitar riesgos innecesarios a edades tempranas. Aunado a esto, el grupo de estudiantes encuestados de noveno también comparte opiniones en cuanto a la inadecuada formación que se ha dado, ya que centran sus necesidades de formación y conocimientos al tener y no al ser; de modo que para estos estudiantes la sexualidad debe entenderse como:

(...) un elemento básico de la personalidad; un modo propio de ser, de manifestarse, de comunicarse con los otros, de sentir, expresar y vivir el amor humano. Se visualiza como una fuente de placer corporal y espiritual, que sobrepasa las fronteras del fenómeno reproductivo y el ámbito de lo privado, enriqueciéndose en los vínculos interpersonales. (República de Costa Rica, MEP, 2012, p. 4)

\section{Análisis de los resultados de instrumento aplicado a los docentes y las docentes}

En relación con la pregunta sobre las posibles estrategias metodológicas que implementarían para abordar los temas que se presentan en el programa EASIE, los sujetos entrevistados mencionaron variadas estrategias, entre ellas: medios audiovisuales (presentaciones de powerpoint, videos, grabaciones); dependen del tópico que se desarrolle, ya que los contenidos de los programas deben ser trabajados transversalmente dentro de una programación establecida; estrategias vivenciales; metodologías inclusivas con el fin de que el 
estudiante y la estudiante sientan la confianza para aprender e informarse adecuadamente, por último se indicó análisis de situaciones, discusiones de problemas, videos y conferencias para desarrollar dichas clases.

En cuanto a la efectividad para lograr captar la atención del estudiantado y desarrollar la confianza necesaria para hablar de estos temas sin romper la línea de respeto entre docente-estudiante, las 5 personas (100\%) participantes expusieron, que sí se logra con la utilización de las estrategias anteriormente mencionadas, puesto que el tema por sí solo genera muchas expectativas e intereses en el estudiantado, lo cual permite que se desarrolle ese diálogo entre docente y estudiante, sin embargo, aclaran que hay que dejar claros los límites desde el inicio.

En lo que concierne al dominio del tema, (60\%), el profesorado participante afirma que no presenta un manejo adecuado sobre el tema, ya que siempre hay nuevas tendencias. Además, se indicó que las inquietudes que presenta el estudiantado adolescente no son iguales en todos los casos, es por eso que no se puede decir que se tiene un dominio total del contenido, sino que se debe tomar en cuenta el contexto social que rodea al estudiante y, sobre todo, propiciar una mediación pedagógica que permita aprender para la vida, elemento que se plantea dentro del programa del MEP, al proponer:

(...) equidad de género y se toma en cuenta prioritariamente la promoción del pensamiento crítico y el desarrollo de habilidades, para que el estudiantado pueda construir conductas de protección, no solo durante el ciclo reproductivo, sino en toda su vida, siempre en un marco de respeto a la persona. (República de Costa Rica, MEP, 2012, p. 5)

Además, las 5 personas participantes docentes de ciencias (100\%) mencionaron que el Ministerio de Educación Pública no los ha capacitado para saber cómo tratar dichas temáticas; por lo que consideran de gran importancia recibir capacitaciones para estar actualizados, aclarar y unificar criterios, además, para conocer los lineamientos metodológicos que se proponen.

A propósito de lo recién indicado, se debe resaltar que el estudiantado encuestado manifestó que sus profesores no les dan la suficiente confianza, elemento que se puede relacionar con el grado de conocimiento sobre sexualidad, ya que estas personas docentes dicen tener formación principalmente sobre componentes biológicos y ser autodidactas, mas aducen no tener claridad de cómo deben abordarse los nuevos programas EASIE, puesto que las temáticas son más amplias. 
URL: http://www.una.ac.cr/educare

CORREO: educare@una.cr

Tabla 3

Metodologías y recursos para la implementación del programa educación para la afectividad y sexualidad integral en los estudiantes de secundaria

\begin{tabular}{|c|c|c|c|}
\hline Cuestionante & $\begin{array}{l}\text { Estrategias metodológicas que } \\
\text { implementaría el docente para abarcar } \\
\text { los temas que se presentan en la guía de } \\
\text { afectividad y sexualidad integral }\end{array}$ & $\begin{array}{l}\text { Capacidad de las metodologías } \\
\text { para lograr captar la atención de los } \\
\text { estudiantes y para crear un vínculo de } \\
\text { confianza donde estos puedan abarcar } \\
\text { completamente sus dudas y romper el } \\
\text { limite de relación docente-estudiante }\end{array}$ & $\begin{array}{l}\text { Presencia de un dominio del tema } \\
\text { de sexualidad lo suficientemente } \\
\text { amplio que les permita abarcar } \\
\text { las dudas e inquietudes de los } \\
\text { estudiantes }\end{array}$ \\
\hline $\begin{array}{l}\text { Opinión del } \\
\text { docente } A\end{array}$ & $\begin{array}{l}\text { La normativa y la confrontación para } \\
\text { detectar los conocimientos previos del } \\
\text { estudiante a posteriori, se usaría lecturas } \\
\text { complementarias u otra estrategia, es } \\
\text { todo de acuerdo con el grupo. }\end{array}$ & $\begin{array}{l}\text { Se requiere romper el hielo en esa } \\
\text { relación, esto solo se logra mediante el } \\
\text { diálogo, esto permite establecer una } \\
\text { relación de confianza. }\end{array}$ & $\begin{array}{l}\text { No, porque necesito una } \\
\text { actualización no sobre el tema, sino } \\
\text { sobre la problemática general del } \\
\text { adolecente. }\end{array}$ \\
\hline $\begin{array}{l}\text { Opinión del } \\
\text { docente B }\end{array}$ & $\begin{array}{l}\text { La utilización de otros medios } \\
\text { audiovisuales como presentaciones en } \\
\text { computadoras, videos, grabaciones. }\end{array}$ & $\begin{array}{l}\text { Sí claro, porque eso depende de la } \\
\text { interacción y relación que el docente } \\
\text { pueda crear con sus estudiantes. }\end{array}$ & $\begin{array}{l}\text { No, porque creo que a nivel } \\
\text { general ha faltado asesoramiento } \\
\text { en este camino del MEP hacia } \\
\text { el profesorado, sobre todo en } \\
\text { aspectos no biológicos. }\end{array}$ \\
\hline $\begin{array}{l}\text { Opinión del } \\
\text { docente C }\end{array}$ & $\begin{array}{l}\text { Trabajarlos transversalmente y dentro de } \\
\text { una programación establecida. }\end{array}$ & $\begin{array}{l}\text { De acuerdo en lo vivido en el aula el } \\
\text { tema por si solo crea mucha expectativa } \\
\text { en los alumnos, y genera mucho interés. }\end{array}$ & $\begin{array}{l}\text { No, porque siempre hay aspectos } \\
\text { nuevos que implementar y no se } \\
\text { tiene la verdad absoluta. }\end{array}$ \\
\hline $\begin{array}{l}\text { Opinión del } \\
\text { docente D }\end{array}$ & $\begin{array}{l}\text { Metodologías inclusivas donde el } \\
\text { estudiante sienta la confianza para } \\
\text { aprender e informarse adecuadamente. }\end{array}$ & $\begin{array}{l}\text { Siento que sí es posible siempre y } \\
\text { cuando existan límites claros desde el } \\
\text { inicio. }\end{array}$ & $\begin{array}{l}\text { Sí, porque existe la posibilidad de } \\
\text { informarse si fuese necesario. }\end{array}$ \\
\hline $\begin{array}{l}\text { Opinión del } \\
\text { docente } E\end{array}$ & $\begin{array}{l}\text { Mediante análisis de situaciones, } \\
\text { discusión de problemas, videos y } \\
\text { conferencias. }\end{array}$ & $\begin{array}{l}\text { Sí porque una metodología por muy } \\
\text { atractiva que fuera es un abanico de } \\
\text { posibilidades. }\end{array}$ & Sí, por mi condición autodidacta. \\
\hline
\end{tabular}

Nota: Resultados obtenidos de las entrevistas aplicadas a cinco docentes de secundaria.

\section{Conclusiones}

Por medio de los resultados obtenidos y su respectivo análisis, se pueden rescatar las siguientes conclusiones propias de esta investigación y de la muestra estudiada, por lo que no pueden ser generalizadas a otras poblaciones.

El nivel de conocimiento que posee la mayoría de docentes participantes para el abordaje de los temas de educación sexual, a la luz de los programas sugeridos por el MEP, es bajo y está centrado en el componente biológico propio de la carrera Enseñanza de las Ciencias, razón por la cual solicitan actualización y capacitación para desarrollar un mejor proceso de mediación pedagógica acorde con los nuevos tópicos planteados.

El grado de conocimiento que tiene el grupo de estudiantes de sétimo y noveno nivel que participó en el estudio en cuanto a la temática, se centra en el tener, al referir temas como infecciones de transmisión sexual, método anticonceptivos, y dejan de lado elementos del ser, tales como conocerse, valorarse, entre otros. 
Pese a lo anterior, el estudiantado está anuente a ampliar sus conocimientos en sexualidad.

La mayor parte del estudiantado considera que la implementación de las guías de educación para la afectividad y la sexualidad integral son importantes, ya que es una forma en la que se pueden informar, además de que representan un espacio para construir herramientas sobre dichos contenidos.

Para el estudiantado, la propuesta con la que se abordaba la educación sexual en el MEP, al momento de la investigación, es anacrónica y ambigua, centrada en lo que se debe hacer a la luz de la sociedad y no desde la perspectiva de desarrollo integral del ser humano.

El modelo de enseñanza que los estudiantes de sétimo año prefieren para el desarrollo de la temática de educación sexual en su proceso de aprendizaje fue un modelo más tradicional, ya que estos prefieren que el docente asuma la función de exponer de manera progresiva los conocimientos. En contraste, los estudiantes participantes de noveno año prefieren modelos más vivenciales, tales como el modelo por conflicto cognitivo y por investigación, puesto que prefieren una organización de métodos de apoyo que permitan construir su propio conocimiento a partir de vivencias de otros adolescentes, películas, estudios de caso, entre otros.

En cuanto a las estrategias metodológicas para el abordaje de temas de educación sexual en estudiantes de secundaria, el grupo de docentes afirmó que utilizaría técnicas como: charlas, presentaciones de powerpoint, juegos, entre otros. Además, indicaron que propondrían estudios de caso, de manera que se aprenda desde lo inmediato.

\section{Referencias}

Arce, P., León, A. T., Fleming, I., Morales, M. E., Solano, G. y Vicente, R. (2009). Plan nacional de educación para la sexualidad. Ministerio de Educación Pública, Dirección de Desarrollo Curricular. San José, Costa Rica: MEP, UNA, CIDE, Instituto de la Niñez y la adolescencia. Manuscrito inédito.

Barrantes, R. (2010). Investigación. Un camino al conocimiento. Un enfoque cualitativo y cuantitativo. San José, Costa Rica: EUNED.

Cauich, J. (2005). Estudio biográfico-narrativo de los procesos de desarrollo profesional y de asesoramiento del profesorado en el ámbito de la educación sexual en educación secundaria obligatoria. (Tesis doctoral). Universidad de Granada, Nicaragua. Recuperado de http:// hera.ugr.es/tesisugr/15417554.pdf

Coon,D. (2005). Fundamentos depsicología. (10ªd.).México, D. F.:InternationalThomson Editores. Recuperado de http://books.google.com/books?id=nMTpHyd3j3sC\&pg=PA234\&dq=a prendizaje+por+descubrimiento\&hl=es\&ei=iGioTYIkzLPRAdTKxfkl\&sa=X\&oi=book_ result \&ct=result\&resnum $=2 \& v e d=0 C C w Q 6 A E w A T g K \# v=$ onepage $\& q=$ aprendizaje $\% 20$ por\%20descubrimiento\&f=false 
URL: http://www.una.ac.cr/educare

CORREO: educare@una.cr

Díaz, F. y Hernández, G. (2002). Estrategias docentes para un aprendizaje significativo. Una interpretación constructivista. ( $2^{\mathrm{a}}$ ed.). México: McGraw-Hill.

Fallas, M. A. (2012). Modelo biográfico y profesional: Una respuesta de abordaje de la educación afectiva y sexual en Costa Rica. En M. Castro (Comp.), Memoria II Congreso Iberoamericano de Pedagogía: Diversas perspectivas críticas en el siglo XXI (pp. 598-616). Heredia, Costa Rica: CIDE.

Fallas, M. A., Artavia, C. y Gamboa, A. (2012). Educación sexual: Orientadores y orientadoras desde el modelo biográfico y profesional. Revista Electrónica Educare, 16 (Especial), 53-71. Recuprado de http://www.revistas.una.ac.cr/index.php/EDUCARE/issue/view/418

Fernández, A. (2005). Nuevas metodologías docentes. Recuperado de http://www.upm.es/ innovacion/cd/02_formacion/talleres/nuevas_meto_docent/nuevas_metodologias_ docentes_2.pdf

Hernández, R., Fernández, C. y Baptista, P. (2006). Metodología de la investigación (4a ed.). México, Méxic: McGraw-Hill.

Herrera, Á. M. (marzo, 2009). Las estrategias de aprendizaje. Innovación y experiencias educativas, 16. Recuperado de http://www.csi-csif.es/andalucia/modules/mod_ense/revista/pdf/ Numero_16/ANGELA MARIA_HERRERA_2.pdf

Jabif, L. (2007). La docencia universitaria bajo un enfoque de competencias. Orientaciones prácticas para docentes. Chile: Austral.

Joyce, B. y Weil, M. (2002). Modelos de enseñanza. Barcelona: Gedisa.

Morán, L. (diciembre, 2008). Criterios para análisis comparativo de modelos y diseños educativos. Educación y Educadores, 11(2), 139-158. Recuperado de http://www.redalyc. org/articulo.oa?id=83411210

Organización de las Naciones Unidas para la Educación la Ciencia y la Cultura (UNESCO). (2010). Orientaciones técnicas internacionales sobre educación en sexualidad. Un enfoque basado en evidencia orientado a escuelas, docentes y educadores de la salud. Santiago: Autor. Recuperada de http://unesdoc.unesco.org/images/0018/001832/183281s.pdf

Papalia, D. E.,Wendkos, S. y Duskin, R. (2010). Desarrollo humano. (11ª ed.). México: McGraw-Hill.

Pozo, J. I. (1997). Enfoques para la enseñanza de las ciencias. Madrid: Ediciones Morata. Recuperado de http://www.geocities.ws/javi_her/lec_9b.pdf

Ramo, A. (30 de abril de 2008). La información sexual. El Santafesino. Recuperado de http:// www.elsantafesino.com/opinion/2008/04/29/7051 
República de Costa Rica, Ministerio de Educación Pública. (2012). Programa de estudio de "educación para la afectividad y la sexualidad integral". San José, Costa Rica. Recuperado de http://www.nacion.com/MMediaFiles/nacioncom/ba/ba1eb636-2b95-4d9e-b551a7c0eaa1880e.pdf

Rodríguez, W. (1999). El legado de Vygotski y de Piaget a la educación. Revista Latinoamericana de Psicología, 31(3), 477-489. Recuperado de http://www.redalyc.org/pdf/805/80531304. pdf

Ruiz, F. J. (julio-diciembre, 2008). Modelos didácticos para la enseñanza de las ciencias naturales. Revista Latinoamericana de Estudios Educativos, 3(2), 41-60. Recuperado de http://latinoamericana.ucaldas.edu.co/downloads/Latinoamericana3-2_4.pdf

Sarramona,J.(2008). Teoríadelaeducación reflexiónynormativapedagógica.(2ªed.). Barcelona:Ariel. Recuperado de http://books.google.com/books?id=tkyc4FEWDJ8C\&pg=PA256\&dq=apr endizaje+por+descubrimiento\&hl=es\&ei=1 AumTfzFDMX20gG1-rH8CA\&sa=X\&oi=book_ result \&ct=result \&resnum $=9 \& v e d=0 C E w Q 6 A E w C A \# v=$ onepage $\& q=$ aprendizaje $\% 20$ por\%20descubrimiento\&f=false

Tippelt, R. y Lindemann, H.-J. (2001). El método de proyectos. Ministerio de Educación. Gobierno del Salvador. Recuperado de http://www.halinco.de/html/doces/Met-proy APREMAT092001.pdf

Vargas, E. (Comp.). (1997). Metodología de la enseñanza de las ciencias naturales. San José, Costa Rica: EUNED.

Zárate, I. A. (2003). Factores psicosociales familiares asociados a la iniciación sexual en escolares de educación secundaria de Lima Cercado. (Tesis de maestría). Universidad Nacional Mayor de San Marcos. Lima, Perú. Recuperado de http://sisbib.unmsm.edu.pe/bibvirtualdata/tesis/ salud/zarate_li/t_completo.pdf

\section{Cómo citar este artículo en APA:}

León, G., Bolaños, G., Campos, J. y Mejías, F. (2013). Percepción de una muestra de educandos y docentes sobre la implementación del programa educación para la afectividad y la sexualidad integral. Revista Electrónica Educare, 17(2), 145-165. Recuperado de http:// www.revistas.una.ac.cr/index.php/EDUCARE/issue/current

Nota: Para citar este artículo en otros sistemas puede consultar el hipervínculo "Como citar el artículo" en la barra derecha de nuestro sitio web:

http://www.revistas.una.ac.cr/index.php/EDUCARE/index 